\section{KOMPASS}

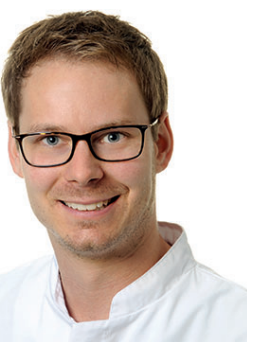

Michael Haas

Medizinische Klinik und Poliklinik III, Klinikum der Universität München, Campus Großhadern, München, Deutschland

\title{
Pankreaskarzinom: Neuerungen in der medikamentösen Therapie
}

Die Therapie des duktalen Adenokarzinoms des Pankreas stellt weiterhin eine große Herausforderung dar. Seit der Einführung von Gemcitabin als Standard-Chemotherapeutikum im Jahr 1997 gab es trotz zahlreicher Therapiestudien nur einen allmählichen, in den letzten Jahren jedoch kontinuierlichen und deutlicheren Fortschritt in der medikamentösen Therapie.

In der adjuvanten, postoperativen Situation konnte zuletzt mit modifiziertem FOLFIRINOX (mFOLFIRINOX) eine deutliche Verbesserung gegenüber der bisherigen Standardbehandlung mit Gemcitabin alleine in sämtlichen Effektivitätsendpunkten wie erkrankungsfreies Überleben, Anteil des erkrankungsfreien Überlebens nach 3 Jahren sowie Gesamtüberleben gezeigt werden. Die Wirksamkeit erstreckte sich hier über nahezu alle Subgruppen, insbesondere auch auf Patienten in höherem Tumorstadium (T3 bzw. T4-Tumoren und R1-resezierte Patienten) [1]. Da mFOLFIRINOX mit einer erhöhten Toxizität verbunden ist, bleibt es allerdings (biologisch) jüngeren Patienten vorbehalten, darf in dieser Gruppe jedoch als neuer Therapiestandard angesehen werden.

Die in der palliativen Behandlung beobachteten Ansprechraten auf effektivere Chemotherapie Kombinationen wie FOLFIRINOX bzw. Gemcitabin+nab-Paclitaxel von 31,6\% bzw. 23,0\% führten zur Initiierung zahlreicher Studien zur neoadjuvanten Therapie bei lokal fortgeschrittenen bzw. borderline-resektablen Pankreaskarzinomen.
Ziel einer neoadjuvanten Behandlung ist die Konversion in einen chirurgisch resektablen Befund bzw. die Erhöhung der Rate an R0-Resektionen. Ein Standard für ein einheitliches Regime existiert nach wie vor nicht. Auf dem ESMO-Kongress 2019 wurden erste Daten der Phase II «NEOLAP-Studie» der Arbeitsgemeinschaft Internistische Onkologie (AIO) präsentiert. Hier wurden 165 Patienten mit unbehandelten, nicht resektablen Pankreaskarzinomen einer Induktionstherapiemit2Zyklen Gemcitabin+nabPaclitaxel unterzogen. Blieben Progress oder Unverträglichkeit aus, erfolgte die Behandlung randomisiert mit 2 weiteren Zyklen Gemcitabin+nab-Paclitaxel vs. 4 Zyklen FOLFIRINOX. Bei mindestens Stabilisierung der Erkrankung war obligat eine explorative Laparotomie vorgesehen.

Die Konversionsrate (komplette, makroskopische Resektion = R0 bzw. R1) betrug im Arm mit Gemcitabin+nab-Paclitaxel 30,6\% gegenüber 45,0\% für Gemcitabin+nab-Paclitaxel gefolgt von FOLFIRINOX (Odds ratio $0,54, p=0,135)$. Der Unterschied in der Konversionsrate zwischen beiden Armen war damit nicht signifikant bei allerdings numerischem Vorteil für die FOLFIRINOX-haltige Induktionstherapie. Eine Konversionsrate von 45\% bei einem Anteil von 74\% R0-Resektionen im Arm mit FOLFIRINOX ist jedoch in jedem Fall ermutigend. Zudem bestand im Gesamtkollektiv ein deutlicher Überlebensvorteil für die Patienten, bei denen die Konversion in einen resektablen Befund erreicht

\section{KARGER}

Fax +497614520714 information@karger.com www.karger.com
() 2019 S. Karger GmbH, Freiburg
PD Dr. Michael Haa

Medizinische Klinik und Poliklinik III

Klinikum der Universität München, Campus Großhader

Marchioninistraße 15, 81377 München

Michael.Haas@med.uni-muenchen.de 
werden konnte (27,4 vs. 14,2 Monate, HR 0,45; $p=0,0035)$, so dass eine explorative Laparotomie nach neoadjuvanter Behandlung stets angestrebt werden sollte [2].

In der Therapie des metastasierten Pankreaskarzinoms wurden auf dem ASCO 2019 erste Daten der POLO-Studie vorgestellt und zeitgleich publiziert. In dieser Phase-IIIStudie wurden Patienten mit nachgewiesener Keimbahnmutation im BRCA1/BRCA2Gen randomisiert einer Erhaltungstherapie mit dem Poly(ADP-Diphosphat-Ribose) Polymerase(PARP)-Inhibitor Olaparib vs. Placebo unterzogen. Alle eingeschlossenen Patienten hatten vorher mindestens eine Erkrankungsstabilisierung nach 16 Wochen unter einer platinbasierter Erstlinienchemotherapie erreicht. Von 3315 gescreenten Patienten wiesen 247 (7,5\%) eine
Keimbahnmutation im BRCA1/2-Gen auf. Das progressionsfreie Intervall war für Olaparib signifikant verlängert (7,4 vs. 3,8 Monate, HR 0,82; $p=0,004)$. In einer Zwischenanalyse für das Gesamtüberleben bestand mit 18,9 vs. 18,1 Monate (HR 0,91; $p=0,68$ ) kein signifikanter Unterschied [3]). Die endgültigen Ergebnisse sind hier sicherlich abzuwarten, jedoch könnten erstmalig in der Behandlung des Pankreaskarzinoms molekular definierte Subgruppen mit Nachweis von Defekten in DNA-Reparaturgenen die Möglichkeit für eine personalisierte Therapie bieten.

\section{Zusammenfassung}

In der adjuvanten Behandlung hat sich die beim metastasierten Pankreaskarzinom bekannte überlegene Wirksamkeit von FOLFI-
RINOX vs. Gemcitabin in Form des modifizierten FOLFIRINOX-Regimes bestätigt. Auch in der neoadjuvanten Therapie von lokal fortgeschrittenen bzw. borderline-resektablen Pankreaskarzinomen bieten die effektiveren Kombinationstherapien Gemcitabin+nab-Paclitaxel bzw. FOLFIRINOX die Chance auf eine Konversion in einen resektablen Befund. Die Ergebnisse der POLO-Studie lassen erstmalig auf eine zielgerichtete Behandlung für molekular definierte Subgruppen beim Pankreaskarzinom hoffen.

Eine spannende Lektüre wünscht Ihnen

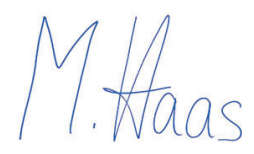

Ihr Michael Haas

\section{Literatur}

$\checkmark 1$ Conroy T, Hammel P, Hebbar M et al. FOLFIRINOX or gemcitabine as adjuvant therapy for pancreatic cancer. New Engl J Med 2018; 379:2395.

2 Kunzmann V, Algül H, Goekkurt E et al. Conversion rate in locally advanced pancreatic cancer (LAPC) after nab-Paclitaxel/Gem- citabine- or FOLFIRINOX-based induction chemotherapy (NEOLAP) - Final Results of a multicenter randomised Phase 2 AIO trial. ESMO Congress 2019, \#671O.

3 Golan T, Hammel P, Reni M et al. Maintenance olaparib for germline BRCA-mutated pancreatic cancer. New Engl J Med 2019;381:317. 